\title{
Towards a Miniaturized Device to Evaluate the BOD Parameter of Wastwater
}

\author{
L. Recoules, S. Jouanneau, G. Thouand, A. M. Gue and A. Boukabache
}

\begin{abstract}
The concentration of organic pollutants present in wastewater is classically evaluated by measuring the quantity of dissolved oxygen during five days; it is quantified by the so called BOD5 parameter (Biological Oxygen Demand). This work constitutes the first step of an overall strategy targeting to improve the monitoring of this indicator of the water quality. We focus on the development of a microsystem approach allowing monitoring the $\mathrm{O}_{2}$ consumption induced by the biodegradation process of organic matter. To evaluate the organic pollutants concentration, we use Escherichia coli as bacterial indicator, confined in a PDMS-glass chip. Their metabolic activity in presence of organic molecules is deduced from their oxygen consumption. These measurements are ensured by optical sensors present in each of the five instrumented chambers of the chip. The results show that the microsystem approach is suitable to measure simultaneously different concentrations of organic load, and that it is possible to reduce the analysis time. By examining the $\mathrm{O}_{2}$ diffusion towards the walls of the device, we analyze the different part of the experimental results; it allows, on the basis of a theoretical model, to predict a precise evaluation of the BOD value within few hours.
\end{abstract}

Index Terms-BOD, wastewater, bacteria, PDMS chip, optods.

\section{INTRODUCTION}

In the wastewater treatment plants (WWTP), one of the most important tests to determine the water quality is the parameter called Biological Oxygen Demand (BOD5): it is based on the evaluation of the oxygen $\left(\mathrm{O}_{2}\right)$ quantity used by bacteria to degrade the organic molecules present in a representative water sample [1], [2]. The normalization of this approach imposes duration of five days for measurement, by comparing the oxygen quantities consumed on this period [2]. The drawbacks of this approach are multiple: stationary apparatus; non differentiation between organic molecules, too long measurement duration, etc...

If we keep the same principle based on the use of bacteria as an indirect transducer, there are needs for the development of micro-devices, capable to give more precise indications about the water quality within a reasonable time. To that purpose, by associating microsystems technology and biological techniques, we have initiated a new approach based on an instrumented microfluidic microsystem

Manuscript received October 9, 2018; revised April 12, 2019. This work was supported in part by the ANR Agency and CNRS (France)

L. Recoules is with LETI, Grenoble, France (e-mail: loic.recoules@gmail.com).

S. Jouanneau and G. Thouand are with University of Nantes, France (e-mail:

gerald.thouand@univ-nantes.fr).

A. M. Gue and A. Boukabache are with LAAS-CNRS, France (e-mail:gue@laas.fr, aboukaba@laas.fr). integrating five separated compartments and used as micro-laboratories [3]. Hence, the monitoring of the oxygen consumption of different and selected bacteria immobilized in adjacent micro-wells should give complementary information about the load in organic matter of the water sample. In order to realize quick prototyping, we have designed and implemented PDMS-Glass chips. To overcome some unexpected aspects of the manipulation of 'in vivo' sensors, i.e. bacteria, we have limited our experiments to a unique type of bacteria, E.coli. As perspective, we planned to use other specialized bacteria.

\section{BOD PRINCIPLES AND MICROSYSTEM CHIP}

The aerobic biodegradation consists to oxidize the organic matter by biological processes. The biochemical reaction, summarized in equation (1), needs the presence of microorganisms [4] in the water sample to transform the carbonaceous matter $\mathrm{C}$, through the dissolved oxygen $\mathrm{O}_{2}$, in a biomass and molecules $\mathrm{CO}_{2}$ and $\mathrm{H}_{2} \mathrm{O}$ :

$$
X_{i}+\mathrm{O}_{2}+\mathrm{C} \quad \rightarrow \quad X_{f}+\mathrm{CO}_{2}+\mathrm{H}_{2} \mathrm{O}
$$

where $X_{i}$ and $X_{f}$ are the initial and final population of bacteria.

Examination of this equation shows that, for assessing the parameter BOD, it is possible to use either measurements of the concentrations of $\mathrm{O}_{2}$ consumed by bacteria or that of the $\mathrm{CO}_{2}$ produced by the biodegradation reaction. The first one is the most used by the water operators: it has the advantage to be direct.

The BOD value is obtained with more or less accuracy, depending on the sophistication of the methods and equipment used [5]. The BOD5 reference (ISO 5815) is hence calculated through two values of $\mathrm{O}_{2}$ concentration: one, at $t=0$, and the other after 5 days.

Many research approaches have been developed either to measure the BOD5 parameter or to predict it, using different transduction methods to convert the concentration of oxygen towards an electrical or optical signal [2,] [5], [6]. None of them is entirely satisfactory because they need sophisticated equipment, long time analysis, or are affected by important measurements uncertainties [2], [7]. In addition to these technological aspects, it appears that two biological strategies are opposed. The first one, based on microbial population (recommended by the reference ISO 5815), has the advantage to provide a pertinent information, but time and space varying. On the opposite, the second one uses only one strain to assess the BOD parameter. In this case, the information lacks of representativeness but is very reproducible. To overcome the limitations of these two strategies, an approach 
based on the use of a set of known and controlled strains seems to be a relevant alternative.

By introducing the microsystem technology and by adapting it to the micro biology field, we have developed an original approach to obtain a micro-device capable to give a new dimension to the measurement of pollutants through the use of bacteria [8]. By adopting a multi sensing approach, our principal objective has been to develop an experimental protocol to follow-up the $\mathrm{O}_{2}$ quantity present in each of several instrumented micro-wells. These measurements could be carried out in a reasonable period of time, allowing afterwards assessing the load of organic pollutants in the analyzed samples.

Based on a PDMS/Glass technology [8], we have designed and fabricated chips having five wells to test different bacteria strains or, for a given bacteria, different nutrients concentrations [3]. Each of these micro chambers is equipped with optical sensor 'optod' whose fluorescence intensity changes with the quantity of oxygen present in the water sample [3].

In Fig. 1 are reported the principals steps to fabricate the chips having micro-wells and oxygen sensors.

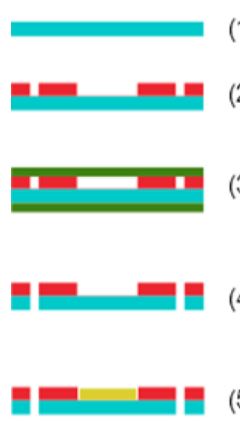

A

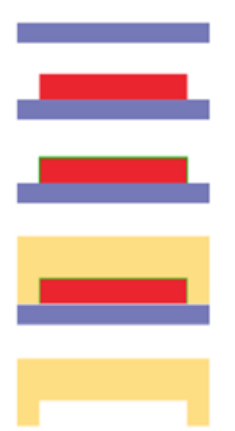

B

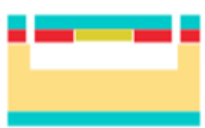

C

Fig. 1. Description of the technological process to realize a bioMEMS chip: $\mathrm{A}$ and $\mathrm{B}$ are the two parts of the microsystem; $\mathrm{C}$ represents the complete microchip, equipped with 'optode' sensors.

The main steps to realize the two parts A and B involve some microelectronic techniques: each of them requires the use of the SU8 epoxy resin either as a dry film or as a PDMS mold.

1) For the top part constituting the cover (A):

- Cleaning of a glass wafer;

- SU8 spin-coating and photolithography;

- Lamination of a dry SU8 film;

- drilling of holes and removal of the SU8 film;

- optode bonding.

2) For the lower part constituting the microfluidic network and containing wells and channels (B):

- Cleaning of a silicon wafer;

- SU8 spin-coating and photolithography according to the dimensions of the micro-chambers;

- surface protection by a thin layer film of Octadecyltrichlorosilane (OTS);

- PDMS molding and curing;
- PDMS film release.

As it can be seen, this fabrication process is adapted to use optode as oxygen sensors. Knowing that it is a solid device, its integration into the MEMS needs to glue it on the glass cover.

The tightness of the device with optode has been performed simply by a pressure action. Indeed, the cover and the PDMS structure are placed on a mechanical support, which allows the two parts to press the one against the other. The deformability of the PDMS ensures then the fluidic tightness.

A detailed view, accompanied by photography, of the final microsystem ready to use is represented in Fig. 2.

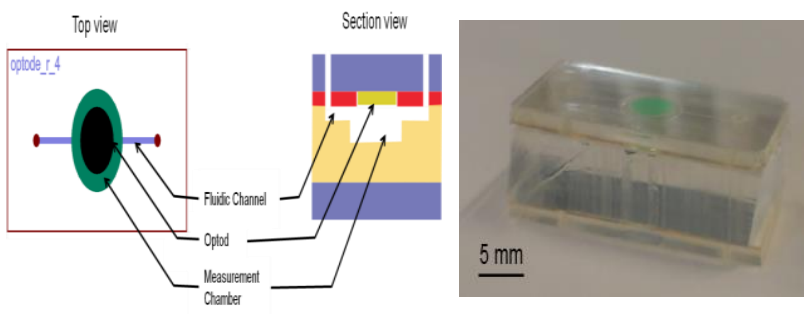

Fig. 2. Schematic representation of a one-well instrumented microfluidic device and the photography of a complete microsystem ready to use.

The device used for experiments contains five wells, each of them having a volume capacity of $\sim 20 \mu \mathrm{L}$, and is provided for receiving one bacteria type or a predefined pollutant.

Knowing that the PDMS is partially permeable to gas [3], we have coated the walls of the micro-chambers with a SU8 polymer film.

\section{MEASUREMENTS}

The experimental protocol can be summarized as follows: preparation of lyophilized E.coli bacteria; injection of solutions containing living bacteria and standardized nutrients Luria-Bertani (LB buffer) at different concentrations in the micro-chambers maintained at $30^{\circ} \mathrm{C}$. The measurement of bacteria activity is performed with optodes electrically polarized and activated every five minutes, for total measurements duration of 10 hours. Our first tests have been performed to compare the results obtained with our device ('micro') with those obtained by a classical process conducted by biologists ('macro') with their specific material and apparatus. Other experiments have been done to study the effects of different nutrients concentration on a fixed bacteria population.

\section{A. Macro-Micro Comparison}

The dissolved oxygen are measured simultaneously in the two types of device (macro and micro) for a fixed bacteria and nutrients concentration $\left(9.210^{7}\right.$ cells per $\mathrm{mL}$ in $\mathrm{LB}$ buffer). The results reported in Fig. 3 show that there are two remarkable parts, each of them linked to many aspects of the protocol used in the experiments.

When the time measurement is less than three hours, it can observed that there is quasi-perfect concordance between the curves 'micro' and 'macro'; after that, we observe an important divergence of the curves. Thus, we can give some confidence to experiments conducted with our micro-device, while seeking tout understand the source of the difference 
between the two curves in the last part.

\section{B. Experiments with Microsystem Device}

By using a test bench developed in our laboratory [5], we have measured the $\mathrm{O}_{2}$ consumed by a population of $\sim 10^{7}$ cells per $/ \mathrm{mL}$ for five dilutions of LB buffer $(1,1 / 10,1 / 100$, $1 / 300$ and $1 / 500$ ).

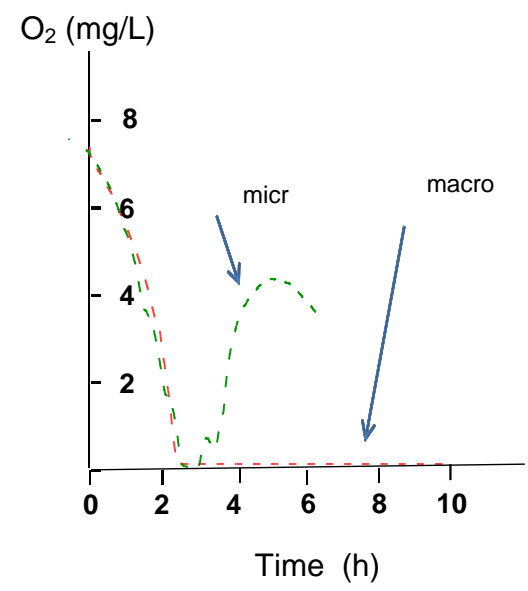

Fig. 3. Experimental curves representing the oxygen consumption by bacteria E.Coli ( $9.210^{7}$ cells per $\mathrm{mL}$ ), in LB buffer, respectively in a ' macro' device and our microsystem chip.

The experimental results for simultaneous measurements are reported in Fig. 4.

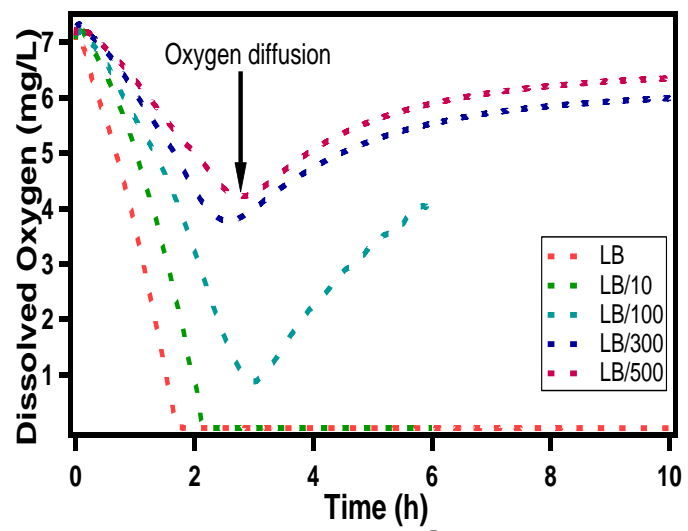

Fig. 4. Oxygen consumption by E.Coli $\left(\sim 10^{7}\right.$ cells per $\left.\mathrm{mL}\right)$ present in a water sample for five dilution of LB buffer ( 5 concentrations of nutrients).

It can be observed that on one side, the quantity of oxygen consumed by bacteria is non-constant, indicating the temporal variability of their metabolism; on the other side, the variations of the $\mathrm{O}_{2}$ concentration in the five chambers, follow a reproducible, but non-identic evolution.

\section{ANALYSIS AND PROSPECTIVE}

The global analysis of results reported on Fig. 3 shows that there are two remarkable zones:

1) The first one shows a regular decrease of oxygen during the period 0 to 3 hours: the observed kinetics vary according to the carbonaceous concentration and present a non-linear rate variation ;

2) The second zone includes two specific cases:

- Either, all nutrients were degraded by cells inducing a stop of the biological oxygen consumption. The $\mathrm{O}_{2}$ concentration in the microchip increases due to a parasitic oxygen contamination (results obtained with $\mathrm{LB} / 100, \mathrm{LB} / 300$ and LB/500).

- Or, the available dissolved $\mathrm{O}_{2}$ concentration is insufficient to oxidize all organic matter. The measured concentration is null (results obtained with LB and $\mathrm{LB} / 10$ ). In this case, the parasitic oxygen allows feeding the biodegradation process by cells.

For a fixed bacteria concentration, the first period can constitute an important indicator to assess the evolution of the BOD: it shows that the bacteria adapt their temporal oxygen consumption to the richness of their living environment. Hence, the tangent attached to these curves will be used as a powerful tool to evaluate the quantity/quality of pollutants present in a water sample.

By examining the topology and the physical properties of the device, we have identified two possible sources of this parasitic oxygen:

- Air imprisoned at the top of the micro wells: when the wells are not entirely filled, their effect is negligible because the $\mathrm{O}_{2}$ quantity is very small and not renewable;

- PDMS properties: knowing that this material is partially permeable to the oxygen gas, we have estimated that the $\mathrm{O}_{2}$ quantities flowing towards the protected walls of the micro chambers imbalance those present in the sample.

This rapid prototyping with PDMS/glass chips confirm, at least for the first hours of the measurements, that it is possible to follow correctly the $\mathrm{O}_{2}$ consumption by bacteria. In the second period, although the cells activity can be differentiated according to their alimentation, the influence of external source of $\mathrm{O}_{2}$ can disturb the results.

For very low nutrients quantity, it can be necessary to extend the measurement duration beyond six hours. For such long time, it becomes necessary to be sure that the chamber has a total tightness. To that purpose, it can be suggested to increase the SU8 coating of the wells; or to adapt the PDMS chips to a resazurin protocol; or to use other microsystem material like SU8 or DF.

\section{THEORY-EXPERIENCE COMPARISON}

To understand what reaction occurs in the chambers of the bioMEMS devices between bacteria and their living environment, we have developed a model based on the equation (1) to compare theoretical curves with our experimental results.

By using the Verhust differential equation [9] describing the bacteria behavior, we have obtained the temporal variation of oxygen consumed by bacteria:

$$
\left[O_{2}\right](t)=\left[O_{2}(0)\right]-g \cdot X_{0} \cdot e^{\mu t} \cdot \frac{X_{\max }}{X_{0} \cdot e^{\mu t}+X_{\max }-X_{0}}
$$

where $X_{\max }$ represents the maximal value of the bacteria concentration; $X_{0}$, its initial concentration; $g$, the oxygen consumption by bacteria, for a particular value of organic molecules; and $\mu$, the bacterial growth.

All these bio-physical parameters depend on the carbonaceous richness of the analyzed sample expressed by 
the factor $D[9]$ :

- $X_{\max }(D)=3 \times 10^{11} \times D^{0.9674}$

- $g(D)=3.2111 \times 10^{-8}+4.1213 \times 10^{-9} \times \ln (D)$

- $\mu(D)=0.53238+0.3814 \times 10^{-3} \times D^{-1.1683}$

By using the value $g=6.410^{-10} \mathrm{mg}$ of $\mathrm{O}_{2}$ per bacteria, it can be observed that the bacterial growth is initially managed by $\mu$ and, in the terminal phase of measurements, by its maximum concentration $X_{\max }$.

The BOD5 value, which is the result of the difference between the initial concentration of Oxygen, $\mathrm{O}_{2}(0)$ and its final concentration after 5 days, can be then related directly to the ratio of bacteria concentrations $X_{0} / X_{\max }$, provided that oxygen is always present.

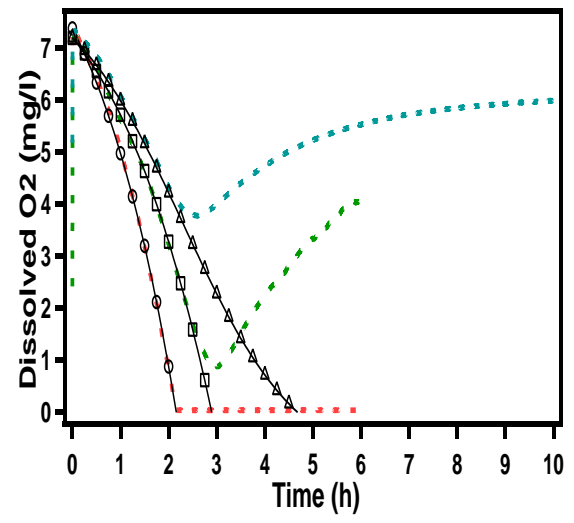

$-\mathrm{LB} / 10$ Experimental
$-\mathrm{L} / 10$ Verhulst model $\rightarrow$ LB/10 Verhulst model 廿 LB/100 Verhulst mode| - - LB/300 Experimental $\triangle \mathrm{LB} / 300$ Verhulst model

Fig. 5. Experimental and theoretical curves of the oxygen consumption by bacteria E. Coli $\left(\sim 10^{8}\right.$ Bact. $\left./ \mathrm{mL}\right)$ positioned in the MEMS micro-chambers in presence of three values of the carbonaceous nutrients LB.

The examination of the curves reported on Fig. 5 shows that there is a good agreement between the experimental values and those obtained from the equation (2), for the period from 0 to 3 hours approximately.

During this time, the bacteria consume the $\mathrm{O}_{2}$ present in water sample, and there is a diminution of the dissolved oxygen present in each well, with a pronounced effect at high concentration of LB.

For the second period, after three hours approximately, it can be observed a mismatch between theory and experience, since the one remain constant while the other grows. It is clear that the experimental process suffer from an undesired source of oxygen or a modified metabolism of bacteria.

\section{CONCLUSION}

To reduce the BOD time measurements in wastewater, we have introduced a microsystem approach by associating bacteria and micro-devices. By using a PDMS-glass technology, we have demonstrated that this miniaturization is suitable, and it opens promising perspectives to enhance many of the indicators of the water quality. By developing a poly-wells instrumented PDMS device, we have demonstrated that it is possible to conduct different measurements either to detect the presence of organic molecules at different concentrations.

By using a PDMS/glass technology, it has been demonstrated that the optical signal, thanks to optodes, allows determining some significant indicators of the water quality: concentration of organic molecules, rate and duration of their consumption etc.... We have conducted some manipulations and experiments which led to conclude that the microsystem approach is suitable to reduce the time measurements of BOD, at least for wastewater containing a lot of organic molecules. This capability responds fully to the multisensorial approach (several microbial strains) of the overall concept of the BOD measurement in which this study is integrated.

It has been demonstrated that, not only the miniaturization associated to the measurements procedure of BOD is possible, but also that the experimental and theoretical results converge towards, at least, a more significant and rapid evaluation.

By associating a theoretical model and a PDMS/glass instrumented micro-device, we demonstrate that it is possible to extract a significant indicator for the BOD value in a short time, less than 3 hours. This allows determining much high carbonaceous loads in a sample.

\section{REFERENCES}

[1] ISO 2003 Water Quality - Determination of Biochemical Oxygen Demand After $n$ Days (BODn) - Part 1: Dilution and Seeding Method with Allylthiourea Addition (Standard International Organization for Standardization) ISO 5815-1, 2003

[2] S. Jouanneau et al., "Methods for assessing biochemical oxygen demand (BOD): A review," Water Res., 2014, vol. 49, pp. 62-82.

[3] L. Recoules et al., "A MEMS approach to determine the biochemical oxygen demand (BOD) of wastewaters," J. Micromech Microeng, 2017, p. 27.

[4] T. Kono, "Kinetics of microbial cell growth," Biotechnology and Bioengineering, vol. 10, no. 12, pp. 105-131, 1968.

[5] J. Wang et al., "An innovative reactor-type biosensor for BOD rapid measurement," Biosensors \& Bioelectronics, 2010, vol. 25, no. 17, pp. 1705-1709.

[6] D. A. Markov et al., "Variation in diffusion of gases through PDMS due to plasma surface treatment and storage conditions," Biomedical Microdevices, vol. 16, no. 1, pp.91-96, 2014.

[7] P. Villalobos et al., "A BOD monitoring disposable reactor with alginate-entrapped bacteria," Bioprocess and Biosystems Engineering, vol. 33, no. 18, pp. 961-970, 2010.

[8] L. Recoules, "Biosensor for monitoring the quality of the water Application to wastswater," $\mathrm{PhD}$ thesis, UPS, Toulouse (France), 2015.

[9] M. Peleg, M. G. Corradini, and M. D. Normand, "The logistic (Verhulst) model for sigmoid microbial," Food Research International, vol. 40, no. 17, pp. 808-818, 2007.

Loic Recoules received his $\mathrm{PhD}$ degree in electronics from the University of Toulouse, France, in 2015. After a post-doctoral position in 3IT (Sherbrooke University, Canada) 2016 - 2017. He is currently engineer R\&D Micro-Nano Systèmes at CEA_LETI (Grenoble, France).

Sulivan Jouanneau received his $\mathrm{PhD}$ degree in process engineering from the University of Nantes, France, in 2011. His thesis is entitled "Development of a data analysis metrology by decision trees applied on a multi-ways biosensor." He is currently assistant professor at the University of Nantes.

His research activities are devoted to the environmental monitoring of biodegradation and ecotoxicology using based-bacteria biotechnologies. He is mainly involved in the development of analytical methods to assess some environmental polluants (specific or not) in the field. These strategies are based on the coupling between microbial bioelements and statistical approaches of data mining and are implemented into automatized platforms (biosensors).

Dr. Jouanneau was notably responsible of the development of the measurement methods in the framework of the project BIOGUARD (funded by the French national research agency) on assessment of the organic load in domestic wastewaters in collaboration with Suez Environnement. Scientific production: 12 International peer review articles, 5 book chapters, 12 International conferences.

Gerald Thouand received his $\mathrm{PhD}$ degree in microbiology from the University of Nancy, France, in 1993. He is tenured professor in 
microbiology at the University of Nantes. Deputy for research innovation at the French Ministry of Research (DRRT Pays de La Loire) and Auditor at the Institute of Higher Studies for Science and Technology (IHEST, Paris).

His research interest includes environmental monitoring of biodegradation and biotechnology using microbial biosystems. He is mainly involved in the development of biosensors for chemical pollutants detection and pathogenic bacteria. He is member of the Society for Applied Microbiology (SFAM, UK) and Member of the scientific committee of the International Society of Bioluminescence and Chemiluminescence. He is Associate editor for Frontiers in Microbiology and editor for Environmental Science and Pollution Research (ESPR, Springer).

He has scientific production: 56 International peer review articles, 4 patents, 16 book chapters or edition, 60 International conferences, $20 \mathrm{PhD}$ students guided.
Anne-Marie Gué studied physics at the National Institute for Applied Science and received her $\mathrm{PhD}$ degree at the University of Toulouse. She joined LAAS-CNRS in 1988 as a CNRS senior scientist. Since 1994, she is involved in the development of microtechnologies and microsystems for chemical and biological applications. She has first been working in the design and fabrication of miniaturized chemical and bio-sensors. Her activity is now focusing on microfluidic aspects. She has been deputy director of LAAS-CNRS from 2011 to 2016.

Ali Boukabache received PhD degree from University of Toulouse (1983). $\mathrm{He}$ has been a teacher/researcher successively at University of Constantine (Algeria) until 2001 and since 2004, University of Toulouse. His research activities are mainly devoted to MEMS and their applications, particularly on microfluidics. 